\title{
Trajectory Smoothing as a Linear Optimal Control Problem
}

\author{
Biswadip Dey \\ Dept. of Electrical and Computer Engg., \& \\ Institute for Systems Research, \\ University of Maryland, \\ College Park, MD 20742, USA. \\ biswadip@umd. edu
}

\author{
P. S. Krishnaprasad \\ Dept. of Electrical and Computer Engg., \& \\ Institute for Systems Research, \\ University of Maryland, \\ College Park, MD 20742, USA. \\ krishna@umd.edu
}

\begin{abstract}
In many areas of science and engineering there is a need for techniques to robustly extract velocity and its derivatives from a finite sample of observed positions. The extracted information can be used to infer related quantities such as curvature and speed, which are important for analysis of strategies and feedback laws associated with the motion. In this work a novel approach is proposed to reconstruct trajectories from a set of discrete observations. A simple linear model is used as the generative model for trajectories, and high values of the jerk (derivative of the acceleration) path integral are penalized during reconstruction. The positions, reconstructed in this way, can be represented as a linear combination of the sample data. The regularization (penalty) parameter plays a very important role in the reconstruction process, and it may be determined from data using ordinary cross validation.
\end{abstract}

\section{INTRODUCTION}

One finds evidence of collective motion in many natural settings. From schools of fish [1] to aerial display by large flocks of starling [2] - we find agents moving collectively by interacting with each other. On the other hand, pursuit can be observed in different kinds of intra- and inter- species encounters, such as bat-bat pursuit [3] or dragonfly foraging [4]. As both collective motion and pursuit play a significant role also in engineering settings, it seems to be a relevant effort to explore underlying strategies and control laws governing collective motion, by extracting parameters of motion (namely curvature, speed, lateral acceleration etc.) from sampled observations of trajectories.

The problem of recovering an underlying smooth signal from sampled noisy observations arises in many fields of applied science. This inverse problem is ill-posed in the sense that naive solutions are highly sensitive to noise, and non-unique. Tackling such problems by the method of regularization has a long history [5]. In this paper we focus on a class of such problems associated to reconstructing smooth trajectories of animal movement (e.g. starlings in a flock [2], echolocating bats engaged in prey capture [3][6], fish schools [1]) from time samples of 3D position. Typically such time-samples are gathered from video imagery (at rates anywhere from $10 \mathrm{~Hz}$ to $1000 \mathrm{~Hz}$ ) using a multi-camera network subject to computer vision algorithms to determine 3D positions of feature points (e.g. center of mass of a starling in flight) from 2D projections. Taking the result of such vision processing as a starting point, we seek to extract noise-robust smooth trajectories. In what follows, we turn this into an optimal control problem for a generative model of feature point trajectories, and the choice of cost functional to be optimized specifies the regularization imposed on the data.

Treating feature points as self steering particles with trajectories $r(t)$ in three dimensions, a natural generative model is

$$
\left.\begin{array}{l}
\dot{g}=\nu g \xi \\
r=\left[\begin{array}{ll}
I & 0
\end{array}\right] g e_{4} .
\end{array}\right\}
$$

Here $I$ denotes the $3 \times 3$ identity matrix, $g(t)$ denotes a $4 \times 4$ matrix representation of a curve in the special euclidean group $S E(3)$ :

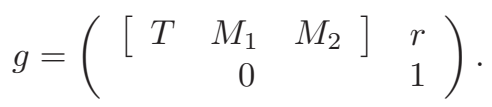

The $3 \times 3$ block [ $\left.\begin{array}{ccc}T & M_{1} & M_{2}\end{array}\right]$ denotes a natural framing of $r$, an orthonormal triad with $T$ the normalized velocity. Letting $\xi_{0}=E_{14} ; \xi_{2}=E_{13}-E_{31} ; \xi_{3}=E_{21}-E_{12}$, denote standard basis elements in se(3) the Lie algebra of $S E(3)$, and $e_{4}$ denote a standard basis vector in $\mathbb{R}^{4}$, we set

$$
\xi=\xi_{0}+u \xi_{3}-v \xi_{2}
$$

Then $u$ and $v$ are natural curvatures (steering controls) and $\nu$ is the speed of the trajectory $r(t)$. The existence and uniqueness (up to a single rotational degree of freedom) of such a generative model for every twice differentiable trajectory $r(t)$ is discussed in [7], and exploited in a series of papers (c.f. [8]) investigating pursuit models in nature and engineering.

This research was supported in part by the Air Force Office of Scientific Research under AFOSR Grant No. FA95501010250, and by the ODDR\&E MURI Program Grant No. N000140710734 (through the Office of Naval Research). 
In this setting the optimal control problem takes the form (here $\lambda>0$ ),

$$
\operatorname{Minimize}\left(\sum_{i=0}^{N}\left\|r\left(t_{i}\right)-r_{i}\right\|^{2}+\lambda \int_{0}^{T}\left(\dot{u}^{2}+\dot{v}^{2}+\dot{\nu}^{2}\right) d t\right)
$$

subject to the generative model (1), given a time sample $\left\{r_{i}\right\}_{i=0}^{N}$. The nonlinear nature of this optimization problem leads one to resort to mathematical programming algorithms as in [9], to determine the inputs $u, v, \nu$. We note that the purpose of such reconstruction is to look for evidence in the data for theoretically based feedback steering control laws (in [9][10] this pertained to sonar-guided pursuit of a prey insect by a bat). In the absence of a full-fledged integrability theory for optimal control problems in Lie groups (but see [11][12]) one resorts to numerical, constrained optimization for (2).

On the other hand, one observes that steering control may be expressed in terms lateral acceleration. This in turn suggests resorting to an alternative linear generative model driven by acceleration as input and a regularization cost given by a quadratic in jerk, the derivative of acceleration. This allows one to fully exploit the integrability theory of linear-quadratic optimal control, given by Riccati equations, as in the rest of this paper. For a different view of exploiting linear optimal control for smooth interpolation see [13].

\section{Problem Statement}

Given a time series of observed positions in three dimensional space, our primary objective is to generate a smooth trajectory to fit these data points. To assure smoothness of the reconstructed trajectory we penalize high values of the jerk path integral. This particular penalty term is very relevant and carries a similar effect as the penalty term considered in (2). Moreover it is very significant in the context of physiological movement, as described in [14] and [15].

Let $\left\{r_{i}\right\}_{i=0}^{N}$ be the set of observed positions. We are interested in finding a trajectory $r:[0, T] \rightarrow \mathbb{R}^{3}\left(t_{0}=0, t_{N}=T\right)$ to minimize the following cost:

$$
\sum_{i=0}^{N}\left\|r\left(t_{i}\right)-r_{i}\right\|^{2}+\lambda \int_{0}^{T}\left\|r^{(3)}(t)\right\|^{2} d t
$$

where $(\cdot)^{(k)}$ implies $k$-th derivative of any function, if it exits. $\lambda>0$ acts as a regularization parameter in the above cost. It forces a balance between the goodness of fit and the smoothness of the trajectory. The trajectory dynamics is given by

$$
\begin{aligned}
\dot{r}(t) & =v(t) \\
\dot{v}(t) & =a(t) \\
\dot{a}(t) & =u(t)
\end{aligned}
$$

where $v, a$ and $u$ represents the velocity, acceleration and jerk respectively. Then the cost can be expressed as:

$$
\sum_{i=0}^{N}\left\|r\left(t_{i}\right)-r_{i}\right\|^{2}+\lambda \int_{0}^{T}\|u(t)\|^{2} d t
$$

Now we define a state $x$ as:

$$
x \triangleq\left[\begin{array}{l}
r \\
v \\
a
\end{array}\right]
$$

and therefore the dynamics of the trajectory $x$ can be represented in a compact form, as

$$
\begin{aligned}
\dot{x}(t) & =A x(t)+B u(t) \\
r(t) & =C x(t)
\end{aligned}
$$

where

$$
A=\left[\begin{array}{lll}
0 & I & 0 \\
0 & 0 & I \\
0 & 0 & 0
\end{array}\right] ; B=\left[\begin{array}{l}
0 \\
0 \\
I
\end{array}\right] ; C=\left[\begin{array}{l}
I \\
0 \\
0
\end{array}\right]^{T}
$$

with $I$ being a $3 \times 3$ identity matrix. It is obvious that (4) is controllable and observable. Now we can pose our smoothing problem as a special case of the following constrained optimization problem:

$$
\begin{array}{cl}
\underset{x\left(t_{0}\right), u}{\operatorname{Minimize}} & J\left(x\left(t_{0}\right), u\right)=\sum_{i=0}^{N}\left\|r\left(t_{i}\right)-r_{i}\right\|^{2}+\lambda \int_{0}^{T} u^{T} u d t \\
\text { subject to } & x\left(t_{0}\right) \in \mathbb{R}^{n}, \\
& u \in \mathcal{U}, \\
& \dot{x}(t)=A x(t)+B u(t), \\
& r(t)=C x(t)
\end{array}
$$


where $\mathcal{U}$ is the space of piecewise continuous functions.

\section{Control Theoretic Approach to Data Smoothing Problem}

We begin by applying in the present setting of data smoothing, a standard tool from the theory of least squares, namely the path independence lemma for trajectories of linear systems [16].

\section{A. Path Independence Lemma and Its Application to Completion of Squares}

Consider the quadratic form $x^{T}(t) K(t) x(t)$, where $K:[0, T] \rightarrow \mathbb{R}^{n \times n}$ is a symmetric matrix-valued function. Then, along (5)

$$
\begin{aligned}
& \int_{t_{i}^{+}}^{t_{i+1}^{-}} d\left(x^{T} K x\right)=\int_{t_{i}^{+}}^{t_{i+1}^{-}}\left(x^{T} K(A x+B u)+(A x+B u)^{T} K x+x^{T} \dot{K} x\right) d t \\
\Rightarrow & \int_{t_{i}^{+}}^{t_{i+1}^{-}}\left[\begin{array}{l}
x \\
u
\end{array}\right]^{T}\left[\begin{array}{cc}
A^{T} K+K A+\dot{K} & K B \\
B^{T} K & 0
\end{array}\right]\left[\begin{array}{l}
x \\
u
\end{array}\right] d t+x^{T}\left(t_{i}\right) K\left(t_{i}^{+}\right) x\left(t_{i}\right)-x^{T}\left(t_{i+1}\right) K\left(t_{i+1}^{-}\right) x\left(t_{i+1}\right)=0 .
\end{aligned}
$$

For brevity of notation explicit time dependence will be dropped wherever doing so does not create any ambiguity. Adding (6) over $\left(t_{0}^{+}, t_{1}^{-}\right),\left(t_{1}^{+}, t_{2}^{-}\right), \cdots,\left(t_{N-1}^{+}, t_{N}^{-}\right)$we obtain

$$
\begin{gathered}
\int_{0}^{T}\left[\begin{array}{l}
x \\
u
\end{array}\right]^{T}\left[\begin{array}{cc}
A^{T} K+K A+\dot{K} & K B \\
B^{T} K & 0
\end{array}\right]\left[\begin{array}{l}
x \\
u
\end{array}\right] d t+x^{T}\left(t_{0}\right) K\left(t_{0}^{-}\right) x\left(t_{0}\right)+\sum_{i=0}^{N} x^{T}\left(t_{i}\right)\left(K\left(t_{i}^{+}\right)-K\left(t_{i}^{-}\right)\right) x\left(t_{i}\right) \\
-x^{T}\left(t_{N}\right) K\left(t_{N}^{+}\right) x\left(t_{N}\right)=0 .
\end{gathered}
$$

As the quantity given by (7) equals to zero for any $u \in \mathcal{U}$ and any $K$ differentiable over $\left(t_{i}^{+}, t_{i+1}^{-}\right) \forall i \in\{0,1,2, \cdots, N-1\}$, a multiple of it can be added to the cost $J\left(x\left(t_{0}\right), u\right)$ without any change. Hence we have,

$$
\begin{array}{r}
J\left(x\left(t_{0}\right), u\right)=\lambda x^{T}\left(t_{0}\right) K\left(t_{0}^{-}\right) x\left(t_{0}\right)-\lambda x^{T}\left(t_{N}\right) K\left(t_{N}^{+}\right) x\left(t_{N}\right)+\sum_{i=0}^{N} x^{T}\left(t_{i}\right)\left[\lambda\left(K\left(t_{i}^{+}\right)-K\left(t_{i}^{-}\right)\right)+C^{T} C\right] x\left(t_{i}\right) \\
+\lambda \int_{0}^{T}\left[\begin{array}{l}
x \\
u
\end{array}\right]^{T}\left[\begin{array}{cc}
A^{T} K+K A+\dot{K} & K B \\
B^{T} K & I
\end{array}\right]\left[\begin{array}{l}
x \\
u
\end{array}\right] d t+\sum_{i=0}^{N}\left(r_{i}^{T} r_{i}-2 x^{T}\left(t_{i}\right) C^{T} r_{i}\right) .
\end{array}
$$

As (8) holds true for any choice of $K$, at this point we make the following assumptions on $K$,

$$
\begin{aligned}
& \dot{K}(t)=-A^{T} K(t)-K(t) A+K(t) B B^{T} K(t), \\
& K\left(t_{N}^{+}\right)=0, \\
& K\left(t_{i}^{+}\right)-K\left(t_{i}^{-}\right)=-\frac{1}{\lambda} C^{T} C .
\end{aligned}
$$

With the assumptions (9), the cost $J\left(x\left(t_{0}\right), u\right)$ can be represented as

$$
J\left(x\left(t_{0}\right), u\right)=\lambda x^{T}\left(t_{0}\right) K\left(t_{0}^{-}\right) x\left(t_{0}\right)+\sum_{i=0}^{N}\left(r_{i}^{T} r_{i}-2 x^{T}\left(t_{i}\right) C^{T} r_{i}\right)+\lambda \int_{0}^{T}\left\|B^{T} K(t) x(t)+u(t)\right\|^{2} d t .
$$

Now consider the linear functional $x^{T}(t) \eta(t)$, where $\eta:[0, T] \rightarrow \mathbb{R}^{n}$ is a vector valued function. Then,

$$
\begin{aligned}
& \int_{t_{i}^{+}}^{t_{i+1}^{-}} d\left(x^{T} \eta\right)=\int_{t_{i}^{+}}^{t_{i+1}^{-}}\left(x^{T} \dot{\eta}+(A x+B u)^{T} \eta\right) d t \\
\Rightarrow & \int_{t_{i}^{+}}^{t_{i+1}^{-}}\left[\begin{array}{l}
x \\
u
\end{array}\right]^{T}\left[\begin{array}{c}
A^{T} \eta+\dot{\eta} \\
B^{T} \eta
\end{array}\right] d t+x^{T}\left(t_{i}\right) \eta\left(t_{i}^{+}\right)-x^{T}\left(t_{i+1}\right) \eta\left(t_{i+1}^{-}\right)=0 .
\end{aligned}
$$

Adding (11) over $\left(t_{0}^{+}, t_{1}^{-}\right),\left(t_{1}^{+}, t_{2}^{-}\right), \cdots,\left(t_{N-1}^{+}, t_{N}^{-}\right)$we obtain

$$
\int_{0}^{T}\left[\begin{array}{l}
x \\
u
\end{array}\right]^{T}\left[\begin{array}{c}
A^{T} \eta+\dot{\eta} \\
B^{T} \eta
\end{array}\right] d t+x^{T}\left(t_{0}\right) \eta\left(t_{0}^{-}\right)+\sum_{i=0}^{N} x^{T}\left(t_{i}\right)\left(\eta\left(t_{i}^{+}\right)-\eta\left(t_{i}^{-}\right)\right)-x^{T}\left(t_{N}\right) \eta\left(t_{N}^{+}\right)=0 .
$$


As the quantity given by (12) equals to zero for any $u \in \mathcal{U}$ and any $\eta$ differentiable over $\left(t_{i}^{+}, t_{i+1}^{-}\right) \forall i \in\{0,1,2, \cdots, N-1\}$, a multiple of it can be added to the cost $J\left(x\left(t_{0}\right), u\right)$ in (10) without any change. Hence we have,

$$
\begin{gathered}
J\left(x\left(t_{0}\right), u\right)=\lambda\left(x^{T}\left(t_{0}\right) K\left(t_{0}^{-}\right) x\left(t_{0}\right)+x^{T}\left(t_{0}\right) \eta\left(t_{0}^{-}\right)\right)+\sum_{i=0}^{N} x^{T}\left(t_{i}\right)\left[\lambda\left(\eta\left(t_{i}^{+}\right)-\eta\left(t_{i}^{-}\right)\right)-2 C^{T} r_{i}\right]-\lambda x^{T}\left(t_{N}\right) \eta\left(t_{N}^{+}\right) \\
+\lambda \int_{0}^{T}\left(\left[\begin{array}{l}
x \\
u
\end{array}\right]^{T}\left[\begin{array}{c}
A^{T} \eta+\dot{\eta} \\
B^{T} \eta
\end{array}\right]+\left\|B^{T} K x+u\right\|^{2}\right) d t+\sum_{i=0}^{N} r_{i}^{T} r_{i} .
\end{gathered}
$$

As (13) holds true for any choice of $\eta$, we make the following assumptions on $\eta$,

$$
\begin{aligned}
& \dot{\eta}(t)=-\left(A^{T}-K(t) B B^{T}\right) \eta(t), \\
& \eta\left(t_{N}^{+}\right)=0, \\
& \eta\left(t_{i}^{+}\right)-\eta\left(t_{i}^{-}\right)=\frac{2}{\lambda} C^{T} r_{i} .
\end{aligned}
$$

With the assumptions (14), the cost $J\left(x\left(t_{0}\right), u\right)$ can be represented as

$$
\begin{gathered}
J\left(x\left(t_{0}\right), u\right)=\lambda\left[x^{T}\left(t_{0}\right) K\left(t_{0}^{-}\right) x\left(t_{0}\right)+x^{T}\left(t_{0}\right) \eta\left(t_{0}^{-}\right)\right]+\lambda \int_{0}^{T}\left\|u(t)+B^{T}\left(K(t) x(t)+\frac{1}{2} \eta(t)\right)\right\|^{2} d t+\sum_{i=0}^{N} r_{i}^{T} r_{i} \\
-\frac{\lambda}{4} \int_{0}^{T}\left\|B^{T} \eta(t)\right\|^{2} d t .
\end{gathered}
$$

From (15) it is clear that by choosing

$$
u(t)=u_{o p t}(t) \triangleq-B^{T}\left(K(t) x(t)+\frac{1}{2} \eta(t)\right)
$$

we have

$$
J\left(x\left(t_{0}\right), u_{\text {opt }}\right)=\lambda\left(x^{T}\left(t_{0}\right) K\left(t_{0}^{-}\right) x\left(t_{0}\right)+x^{T}\left(t_{0}\right) \eta\left(t_{0}^{-}\right)\right)+\sum_{i=0}^{N} r_{i}^{T} r_{i}-\frac{1}{4} \lambda \int_{0}^{T}\left\|B^{T} \eta(t)\right\|^{2} d t .
$$

As $\lambda>0$, it is apparent from (17), that the necessary and sufficient condition for the cost to be minimized is, $u=u_{\text {opt }}$ and $x^{T}\left(t_{0}\right) K\left(t_{0}^{-}\right) x\left(t_{0}\right)+x^{T}\left(t_{0}\right) \eta\left(t_{0}^{-}\right)$be minimized over $x\left(t_{0}\right) \in \mathbb{R}^{n}$.

Therefore,

$$
x_{\text {opt }}\left(t_{0}\right)=\underset{x\left(t_{0}\right) \in \mathbb{R}^{n}}{\arg \min }\left(x^{T}\left(t_{0}\right) K\left(t_{0}^{-}\right) x\left(t_{0}\right)+x^{T}\left(t_{0}\right) \eta\left(t_{0}^{-}\right)\right)
$$

and the optimal initial state satisfies the following condition

$$
\left[K\left(t_{0}^{-}\right)\right] x_{\text {opt }}\left(t_{0}\right)+\frac{1}{2} \eta\left(t_{0}^{-}\right)=0 .
$$

Hence, we have

$$
J_{\text {min }}=J\left(x_{\text {opt }}\left(t_{0}\right), u_{\text {opt }}\right)=\sum_{i=0}^{N} r_{i}^{T} r_{i}-\lambda\left[x_{o p t}^{T}\left(t_{0}\right) K\left(t_{0}^{-}\right) x_{o p t}\left(t_{0}\right)+\frac{1}{4} \int_{0}^{T}\left\|B^{T} \eta(t)\right\|^{2} d t\right] .
$$

From (5), it is clear that

$$
J_{\min } \geq 0
$$

or in other words

$$
x_{o p t}^{T}\left(t_{0}\right) K\left(t_{0}^{-}\right) x_{o p t}\left(t_{0}\right)+\frac{1}{4} \int_{0}^{T}\left\|B^{T} \eta(t)\right\|^{2} d t \leq \frac{1}{\lambda} \sum_{i=0}^{N} r_{i}^{T} r_{i} .
$$

It is of relevance to mention here that a Riccati equation of the form

$$
\begin{aligned}
\dot{K}(t) & =-K(t) A-A^{T} K(t)+K(t) B B^{T} K(t) \\
K(T) & =Q=Q^{T} \geq 0
\end{aligned}
$$

has a symmetric, positive semi-definite solution for any $t \leq T$, represented as

$$
K(t)=e^{-A^{T}(t-T)} Q e^{-A(t-T)}-e^{-A^{T}(t-T)} Q\left[(\mathbb{G}(t, T))^{-1}+Q\right]^{-1} Q e^{-A(t-T)}
$$


where $\mathbb{G}(t, T)$ is a controllability Grammian like quantity and its invertibility is guaranteed by the controllability of the system (5). With assurance on the existence of solution, we can make the following claim on the solution of (9).

Proposition 3.1: The solution of the Riccati equation (9) assumes the form

$$
K\left(t_{i}^{-}\right)=\frac{1}{\lambda} \sum_{k=i}^{N} \Phi_{\Sigma}\left(t_{i}, t_{k}\right) C^{T} C \Phi_{\Sigma}^{T}\left(t_{i}, t_{k}\right)
$$

for any $i \in\{0,1, \cdots, N\}$ where $\Sigma(t)=-\left(A-\frac{1}{2} B B^{T} K(t)\right)^{T}$ and $\Phi_{\Sigma}$ is the transition matrix of $\Sigma$.

Proof: We will use mathematical induction to prove the above claim.

From the boundary and jump conditions in (9) it is obvious that the claim holds true for $i=N$.

Now we assume that it holds true for $i=m+1$, or in other words

$$
K\left(t_{m+1}^{-}\right)=\frac{1}{\lambda} \sum_{k=m+1}^{N} \Phi_{\Sigma}\left(t_{m+1}, t_{k}\right) C^{T} C \Phi_{\Sigma}^{T}\left(t_{m+1}, t_{k}\right) .
$$

Using uniqueness of solution, one can easily verify that

$$
K(t)=\Phi_{\Sigma}\left(t, t_{m+1}\right) K\left(t_{m+1}^{-}\right) \Phi_{\Sigma}^{T}\left(t, t_{m+1}\right)
$$

satisfies the Riccatti differential equation

$$
\dot{K}(t)=-A^{T} K(t)-K(t) A+K(t) B B^{T} K(t)
$$

for any $t \in\left(t_{m}, t_{m+1}\right)$.

Therefore,

$$
\begin{aligned}
K\left(t_{m}^{-}\right) & =K\left(t_{m}^{+}\right)+\frac{1}{\lambda} C^{T} C \\
& =\Phi_{\Sigma}\left(t_{m}, t_{m+1}\right) K\left(t_{m+1}^{-}\right) \Phi_{\Sigma}^{T}\left(t_{m}, t_{m+1}\right)+\frac{1}{\lambda} C^{T} C \\
& =\frac{1}{\lambda} \sum_{k=m+1}^{N} \Phi_{\Sigma}\left(t_{m}, t_{k}\right) C^{T} C \Phi_{\Sigma}^{T}\left(t_{m}, t_{k}\right)+\frac{1}{\lambda} \Phi_{\Sigma}\left(t_{m}, t_{m}\right) C^{T} C \Phi_{\Sigma}^{T}\left(t_{m}, t_{m}\right) \\
& =\frac{1}{\lambda} \sum_{k=m}^{N} \Phi_{\Sigma}\left(t_{m}, t_{k}\right) C^{T} C \Phi_{\Sigma}^{T}\left(t_{m}, t_{k}\right) .
\end{aligned}
$$

Hence the claim is proved, as it holds true for $i=m$.

Now we concentrate on the dynamics of $\eta$ given by (14) and introduce a new time-varying matrix

$$
\tilde{\Sigma}(t)=-\left(A-B B^{T} K(t)\right)^{T} .
$$

Then the dynamics of $\eta$ can be represented as

$$
\dot{\eta}(t)=\tilde{\Sigma}(t) \eta(t)
$$

for any $t \in\left(t_{i}, t_{i+1}\right)$, where $i \in\{0,1, \cdots, N-1\}$. Let $\Phi_{\tilde{\Sigma}}$ be the state-transition matrix for (21). Then we have the following proposition.

Proposition 3.2:

$$
\begin{aligned}
& \eta\left(t_{i}^{+}\right)=-\frac{2}{\lambda} \sum_{k=i+1}^{N} \Phi_{\tilde{\Sigma}}\left(t_{i}, t_{k}\right) C^{T} r_{k} \\
& \eta\left(t_{i}^{-}\right)=-\frac{2}{\lambda} \sum_{k=i}^{N} \Phi_{\tilde{\Sigma}}\left(t_{i}, t_{k}\right) C^{T} r_{k}
\end{aligned}
$$

Proof: We will use mathematical induction to prove the above claim.

From the boundary and jump conditions in (14) it is obvious that the claim holds true for $i=N$ as,

$$
\begin{aligned}
& \eta\left(t_{N}^{+}\right)=0 \\
& \eta\left(t_{N}^{-}\right)=-\frac{2}{\lambda} C^{T} r_{N} .
\end{aligned}
$$

Now we assume that it holds true for $i=m+1$, or in other words

$$
\eta\left(t_{m+1}^{+}\right)=-\frac{2}{\lambda} \sum_{k=m+2}^{N} \Phi_{\tilde{\Sigma}}\left(t_{m+1}, t_{k}\right) C^{T} r_{k}
$$




$$
\eta\left(t_{m+1}^{-}\right)=-\frac{2}{\lambda} \sum_{k=m+1}^{N} \Phi_{\tilde{\Sigma}}\left(t_{m+1}, t_{k}\right) C^{T} r_{k} .
$$

Using the dynamics of $\eta$, given by (21), we have the following relationship

$$
\begin{aligned}
\eta\left(t_{m}^{+}\right) & =\Phi_{\tilde{\Sigma}}\left(t_{m}, t_{m+1}\right) \eta\left(t_{m+1}^{-}\right) \\
& =-\frac{2}{\lambda} \Phi_{\tilde{\Sigma}}\left(t_{m}, t_{m+1}\right) \sum_{k=m+1}^{N} \Phi_{\tilde{\Sigma}}\left(t_{m+1}, t_{k}\right) C^{T} r_{k} \\
& =-\frac{2}{\lambda} \sum_{k=m+1}^{N} \Phi_{\tilde{\Sigma}}\left(t_{m}, t_{k}\right) C^{T} r_{k} .
\end{aligned}
$$

Using the jump condition at $t_{m}$, we obtain

$$
\begin{aligned}
\eta\left(t_{m}^{-}\right) & =\eta\left(t_{m}^{+}\right)-\frac{2}{\lambda} C^{T} r_{m} \\
& =-\frac{2}{\lambda} \sum_{k=m+1}^{N} \Phi_{\tilde{\Sigma}}\left(t_{m}, t_{k}\right) C^{T} r_{k}-\frac{2}{\lambda} \Phi_{\tilde{\Sigma}}\left(t_{m}, t_{m}\right) C^{T} r_{m} \\
& =-\frac{2}{\lambda} \sum_{k=m}^{N} \Phi_{\tilde{\Sigma}}\left(t_{m}, t_{k}\right) C^{T} r_{k} .
\end{aligned}
$$

From (22) and (23) it is clear that the claim holds true for $i=m$.

Hence the claim is proved.

Proposition 3.3: $\left(-\Sigma^{T}, C\right)$ forms an observable pair for the problem of our interest (4).

Proof: $K$ is a symmetric matrix by definition, and hence one can assume the following block structure for $K$,

$$
K(t)=\left[\begin{array}{ccc}
K_{11}(t) & K_{12}(t) & K_{13}(t) \\
K_{12}^{T}(t) & K_{22}(t) & K_{23}(t) \\
K_{13}^{T}(t) & K_{23}^{T}(t) & K_{33}(t)
\end{array}\right]
$$

With this particular structure for $K$, we have the following expression of $\Sigma^{T}(t)$ for the jerk path integral minimization problem,

$$
\Sigma^{T}(t)=\left[\begin{array}{ccc}
0 & -I & 0 \\
0 & 0 & -I \\
\frac{1}{2} K_{13}^{T}(t) & \frac{1}{2} K_{23}^{T}(t) & \frac{1}{2} K_{33}(t)
\end{array}\right] .
$$

Now, for the sake of convenience, we use Silverman-Meadows rank condition [17] to prove our claim. To do so, we define the matrix $Q_{o b v}$ as

$$
Q_{o b v}(t)=\left[\begin{array}{llll}
S_{0}(t) & S_{1}(t) & \cdots & S_{n-1}(t)
\end{array}\right]
$$

where $S_{i}(t)$ 's are computed recursively using

$$
S_{k+1}(t)=-\Sigma(t) S_{k}(t)+\dot{S}_{k}(t), \quad S_{0}(t)=C^{T} .
$$

The $S_{i}(t)$ 's will assume the following form,

$$
S_{0}(t)=\left[\begin{array}{l}
I \\
0 \\
0
\end{array}\right], \quad S_{1}(t)=\left[\begin{array}{c}
0 \\
I \\
0
\end{array}\right], \quad S_{2}(t)=\left[\begin{array}{c}
0 \\
0 \\
I
\end{array}\right], \quad S_{3}(t)=-\frac{1}{2}\left[\begin{array}{c}
K_{13}(t) \\
K_{23}(t) \\
K_{33}(t)
\end{array}\right],
$$

and so on. Hence it can be immediately concluded that the pair $\left(-\Sigma^{T}, C\right)$ is observable as the rank of $Q_{o b v}(t)$ is 9 for any $t \in \mathbb{R}^{+} \cup\{0\}$.

Theorem 3.4: The equation (18) is uniquely solvable for almost any time index set $\left\{t_{i}\right\}_{i=0}^{N}$.

Proof: From proposition 1 we have,

$$
\begin{aligned}
K\left(t_{0}^{-}\right) & =\frac{1}{\lambda} \sum_{k=0}^{N} \Phi_{\Sigma}\left(t_{0}, t_{k}\right) C^{T} C \Phi_{\Sigma}^{T}\left(t_{0}, t_{k}\right) \\
& =\frac{1}{\lambda} \sum_{k=0}^{N} \Phi_{-\Sigma^{T}}^{T}\left(t_{k}, t_{0}\right) C^{T} C \Phi_{-\Sigma^{T}}\left(t_{k}, t_{0}\right)
\end{aligned}
$$




$$
\begin{aligned}
& =\frac{1}{\lambda}\left[\begin{array}{c}
C \\
C \Phi_{-\Sigma^{T}}\left(t_{1}, t_{0}\right) \\
\vdots \\
C \Phi_{-\Sigma^{T}}\left(t_{N}, t_{0}\right)
\end{array}\right]^{T}\left[\begin{array}{c}
C \\
C \Phi_{-\Sigma^{T}}\left(t_{1}, t_{0}\right) \\
\vdots \\
C \Phi_{-\Sigma^{T}}\left(t_{N}, t_{0}\right)
\end{array}\right] \\
& =\frac{1}{\lambda} \mathfrak{C}^{T} \mathfrak{C} .
\end{aligned}
$$

Now we investigate the rank of $\mathfrak{C}$ because the solvability of (18) is equivalent to the fact of $\mathfrak{C}$ having full rank. To do so we consider the following system

$$
\begin{aligned}
\dot{\xi}(t) & =-\Sigma^{T}(t) \xi(t) \\
\gamma(t) & =C \xi(t),
\end{aligned}
$$

which is observable (proposition 3.3). We can easily show that the $j$-th derivative of its output can be represented as

$$
\gamma^{(j)}(t)=S_{j}^{T}(t) \Phi_{-\Sigma^{T}}\left(t, t_{i n i}\right) \xi\left(t_{i n i}\right)
$$

where $S_{j}(t)$ 's are defined in (25).

Let $\xi_{1} \neq \xi_{2}$ be two different choice of initial state $\xi\left(t_{0}\right)$ for the system (26) and $\gamma_{i}(t)$ be its output corresponding to the initial condition $\xi\left(t_{0}\right)=\xi_{i}$. Now we define,

$$
\mathcal{Y}_{i} \triangleq\left[\begin{array}{c}
\gamma_{i}\left(t_{0}\right) \\
\gamma_{i}\left(t_{1}\right) \\
\vdots \\
\gamma_{i}\left(t_{N}\right)
\end{array}\right]=\mathfrak{C} \xi_{i} .
$$

Now we claim that the outputs of (26), corresponding to two different initial conditions $\xi_{1} \neq \xi_{2}$, do not match identically over any interval $\mathbb{T} \subset \mathbb{R}^{+} \cup\{0\}$, or in other words, there is no such interval $\mathbb{T} \subset \mathbb{R}^{+} \cup\{0\}$ such that $\gamma_{1}(t)=\gamma_{2}(t)$ for any $t \in \mathbb{T}$. We can prove our claim by contradiction. Let

$$
C \Phi_{-\Sigma^{T}}\left(t, t_{0}\right) \xi_{1}=C \Phi_{-\Sigma^{T}}\left(t, t_{0}\right) \xi_{2}
$$

for all $t$ belonging to some interval $\mathbb{T}$. Then the derivatives, when they exist, should match for any $t^{*}$ in the interior of $\mathbb{T}$, i.e.

$$
\begin{aligned}
& \left.\frac{d^{j}}{d t^{j}}\left(C \Phi_{-\Sigma^{T}}\left(t, t_{0}\right) \xi_{1}\right)\right|_{t^{*}}=\left.\frac{d^{j}}{d t^{j}}\left(C \Phi_{-\Sigma^{T}}\left(t, t_{0}\right) \xi_{2}\right)\right|_{t^{*}} \\
\Rightarrow & {\left[\begin{array}{c}
S_{0}^{T}(t) \\
S_{1}^{T}(t) \\
\vdots \\
S_{n-1}^{T}(t)
\end{array}\right] \Phi_{-\Sigma^{T}}\left(t^{*}, t_{0}\right) \xi_{1}=\left[\begin{array}{c}
S_{0}^{T}(t) \\
S_{1}^{T}(t) \\
\vdots \\
S_{n-1}^{T}(t)
\end{array}\right] \Phi_{-\Sigma^{T}}\left(t^{*}, t_{0}\right) \xi_{2} } \\
\Rightarrow & Q_{o b v}^{T}(t) \Phi_{-\Sigma^{T}}\left(t^{*}, t_{0}\right)\left(\xi_{1}-\xi_{2}\right)=0 \\
\Rightarrow & \xi_{1}=\xi_{2} .
\end{aligned}
$$

But it contradicts our initial assumption about inequality of $\xi_{1}$ and $\xi_{2}$, thereby proves the claim. Hence $\mathfrak{C} \xi_{1} \neq \mathfrak{C} \xi_{2}$ for almost any time index set $\left\{t_{i}\right\}_{i=0}^{N}$. Therefore $K\left(t_{0}^{-}\right)$is positive definite almost surely because $\mathfrak{C}$ has full rank almost surely. When the rank condition fails, i.e. $\mathfrak{C} \xi_{1}=\mathfrak{C} \xi_{2}$, we can consider an arbitrary close perturbation of the original time index. For any given $\epsilon>0$ we can choose a perturbed time index set $\left\{\tilde{t}_{i}\right\}_{i=0}^{N}$, such that the following conditions holds true,

$$
\begin{gathered}
t_{0}=\tilde{t}_{0}, \\
\text { and, }\left[\begin{array}{c}
C \Phi_{-\Sigma^{T}}\left(\tilde{t}_{1}, \tilde{t}_{0}\right) \\
\vdots \\
C \Phi_{-\Sigma^{T}}\left(\tilde{t}_{N}, \tilde{t}_{0}\right)
\end{array}\right] \text { has full rank. }
\end{gathered}
$$

Therefore (18) can be uniquely solved, for almost any time index set $\left\{t_{i}\right\}_{i=0}^{N}$. 
Following Theorem 3.4 the optimal initial condition can be represented as

$$
\begin{aligned}
x_{\text {opt }}\left(t_{0}\right) & =-\frac{1}{2}\left[K\left(t_{0}^{-}\right)\right]^{-1} \eta\left(t_{0}^{-}\right) \\
& =\frac{1}{\lambda}\left[K\left(t_{0}^{-}\right)\right]^{-1} \sum_{k=0}^{N} \Phi_{\tilde{\Sigma}}\left(t_{0}, t_{k}\right) C^{T} r_{k} .
\end{aligned}
$$

\section{B. Linear Structure in The Reconstructed Trajectory}

Under the action of optimal control input $u_{\text {opt }}$ the dynamics of the system of our interest, given in (4), looks like

$$
\begin{aligned}
\dot{x}(t) & =A x(t)+B u_{o p t}(t) \\
& =\left[A-B B^{T} K(t)\right] x(t)-\frac{1}{2} B B^{T} \eta(t) \\
& =-\tilde{\Sigma}^{T}(t) x(t)-\frac{1}{2} B B^{T} \eta(t),
\end{aligned}
$$

or in other words it can be viewed as a time-varying linear system with $\eta$ being the input. Then we can express $x(t)$ as

$$
\begin{aligned}
x(t) & =\Phi_{-\tilde{\Sigma}^{T}}\left(t, t_{0}\right) x_{o p t}\left(t_{0}\right)-\frac{1}{2} \int_{t_{0}}^{t} \Phi_{-\tilde{\Sigma}^{T}}(t, \sigma) B B^{T} \eta(\sigma) d \sigma \\
& =\Phi_{\tilde{\Sigma}}^{T}\left(t_{0}, t\right) x_{o p t}\left(t_{0}\right)-\frac{1}{2} \int_{t_{0}}^{t} \Phi_{\tilde{\Sigma}}^{T}(\sigma, t) B B^{T} \eta(\sigma) d \sigma .
\end{aligned}
$$

It is quite clear from (27) that the optimal initial condition is linear in terms of the observed data points. From (29) we obtain the following representation for $x\left(t_{k}\right)$,

$$
\begin{aligned}
x\left(t_{k}\right) & =\Phi_{\tilde{\Sigma}}^{T}\left(t_{0}, t_{k}\right) x_{o p t}\left(t_{0}\right)-\frac{1}{2} \int_{t_{0}}^{t_{k}} \Phi_{\tilde{\Sigma}}^{T}\left(\sigma, t_{k}\right) B B^{T} \eta(\sigma) d \sigma \\
& =\Phi_{\tilde{\Sigma}}^{T}\left(t_{0}, t_{k}\right) x_{o p t}\left(t_{0}\right)-\frac{1}{2} \sum_{i=1}^{k}\left[\int_{t_{i-1}}^{t_{i}} \Phi_{\tilde{\Sigma}}^{T}\left(\sigma, t_{k}\right) B B^{T} \eta(\sigma) d \sigma\right] \\
& =\Phi_{\tilde{\Sigma}}^{T}\left(t_{0}, t_{k}\right) x_{o p t}\left(t_{0}\right)-\frac{1}{2} \sum_{i=1}^{k}\left[\int_{t_{i-1}}^{t_{i}} \Phi_{\tilde{\Sigma}}^{T}\left(\sigma, t_{k}\right) B B^{T} \Phi_{\tilde{\Sigma}}\left(\sigma, t_{i}\right) \eta\left(t_{i}^{-}\right) d \sigma\right] \\
& =\Phi_{\tilde{\Sigma}}^{T}\left(t_{0}, t_{k}\right) x_{o p t}\left(t_{0}\right)-\frac{1}{2} \sum_{i=1}^{k}\left[\int_{t_{i-1}}^{t_{i}} \Phi_{\tilde{\Sigma}}^{T}\left(\sigma, t_{k}\right) B B^{T} \Phi_{\tilde{\Sigma}}\left(\sigma, t_{i}\right) d \sigma\right] \eta\left(t_{i}^{-}\right) \\
& =\Phi_{\tilde{\Sigma}}^{T}\left(t_{0}, t_{k}\right) x_{o p t}\left(t_{0}\right)+\frac{1}{\lambda} \sum_{i=1}^{k}\left[\int_{t_{i-1}}^{t_{i}} \Phi_{\tilde{\Sigma}}^{T}\left(\sigma, t_{k}\right) B B^{T} \Phi_{\tilde{\Sigma}}\left(\sigma, t_{i}\right) d \sigma \times\left(\sum_{j=i}^{N} \Phi_{\tilde{\Sigma}}\left(t_{i}, t_{j}\right) C^{T} r_{j}\right)\right] \\
& =\Phi_{\tilde{\Sigma}}^{T}\left(t_{0}, t_{k}\right) x_{o p t}\left(t_{0}\right)+\frac{1}{\lambda} \sum_{i=1}^{N}\left[\sum_{j=1}^{\min \{i, k\}}\left(\int_{t_{j-1}}^{t_{j}} \Phi_{\tilde{\Sigma}}^{T}\left(\sigma, t_{k}\right) B B^{T} \Phi_{\tilde{\Sigma}}\left(\sigma, t_{j}\right) d \sigma\right) \times \Phi_{\tilde{\Sigma}}\left(t_{j}, t_{i}\right)\right] C^{T} r_{i} .
\end{aligned}
$$

Therefore the smoothed position at time $t_{k}$ can be expressed as

$$
r\left(t_{k}\right)=\frac{1}{\lambda} \sum_{i=0}^{N}\left[C \mathcal{F}_{\lambda}(k, i) C^{T}\right] r_{i}
$$

where

$$
\mathcal{F}_{\lambda}(k, i)=\Phi_{\tilde{\Sigma}}^{T}\left(t_{0}, t_{k}\right)\left[K\left(t_{0}^{-}\right)\right]^{-1} \Phi_{\tilde{\Sigma}}\left(t_{0}, t_{i}\right)+\sum_{j=1}^{\min \{i, k\}}\left(\int_{t_{j-1}}^{t_{j}} \Phi_{\tilde{\Sigma}}^{T}\left(\sigma, t_{k}\right) B B^{T} \Phi_{\tilde{\Sigma}}\left(\sigma, t_{i}\right) d \sigma\right)
$$

for $i \in\{0,1, \cdots, N\}$.

From (30) it can be concluded that the smoothed outputs are linear combinations of observed data.

Remark 3.5: As $\mathcal{F}(k, i)$ depends only on the sampling time instances, namely $t_{0}, \cdots, t_{N}$, these coefficients can be precomputed.

Remark 3.6: This approach can also be viewed as a global alternative to Savitzky-Golay smoothing filters [18][19], wherein the filtered outputs are obtained by fitting a least square polynomial (locally) through the observed data points. In our approach the local nature is absent instead each of the filtered outputs depends on the complete data set. But because of this global 
nature our approach has its own drawback. This method, in its true form, cannot be used in real-time as it requires all the observations together.

Remark 3.7: The significance of the word "smoothing" is twofold in this context. Firstly this approach penalizes high values of jerk path integral and thereby yields a smoothened trajectory. Moreover, it uses data from both past and future to estimate the present position and thus justifies the usage of "smoothing" in estimation context.

Remark 3.8: The formulation of the problem is an example of fixed interval smoothing. One can use this as a building block and proceed to obtain a fixed lag smoothing algorithm. The path is quite intuitive.

\section{An Alternative Co-State Based Approach}

Here we represent the solution in terms of co-state variables, defined as,

$$
p(t) \triangleq K(t) x(t)+\frac{1}{2} \eta(t)
$$

Then the optimal control input (16) and system dynamics (4) will have the form

$$
\begin{aligned}
u_{\text {opt }}(t) & =-B^{T} p(t) \\
\dot{x}(t) & =A x(t)-B B^{T} p(t)
\end{aligned}
$$

and the dynamics of the co-states will be governed by the following equation

$$
\dot{p}(t)=\dot{K}(t) x(t)+K(t) \dot{x}(t)+\frac{1}{2} \dot{\eta}(t)=-A^{T} p(t) .
$$

Therefore the optimal trajectory between two observation times can be viewed as the base integral curve of the following system

$$
\frac{d}{d t}\left[\begin{array}{l}
x(t) \\
p(t)
\end{array}\right]=\left[\begin{array}{cc}
A & -B B^{T} \\
0 & -A^{T}
\end{array}\right]\left[\begin{array}{l}
x(t) \\
p(t)
\end{array}\right] .
$$

From (33) it is apparent that the dynamics of $p$ is decoupled from that of $x$.

Now we'll focus on the jump conditions for the co-states

$$
\begin{aligned}
p\left(t_{i}^{+}\right)-p\left(t_{i}^{-}\right) & =\left[K\left(t_{i}^{+}\right)-K\left(t_{i}^{-}\right)\right] x\left(t_{i}\right)+\frac{1}{2}\left[\eta\left(t_{i}^{+}\right)-\eta\left(t_{i}^{-}\right)\right] \\
& =\frac{1}{\lambda} C^{T}\left(r_{i}-r\left(t_{i}\right)\right) .
\end{aligned}
$$

We also have the following terminal condition

$$
p\left(t_{N}^{+}\right)=K\left(t_{N}^{+}\right) x\left(t_{N}\right)+\frac{1}{2} \eta\left(t_{N}^{+}\right)=0
$$

as both $K\left(t_{N}^{+}\right)$and $\eta\left(t_{N}^{+}\right)$are equal to zero and by choosing $x\left(t_{0}\right)=x_{\text {opt }}\left(t_{0}\right)$ we get,

$$
p\left(t_{0}^{-}\right)=K\left(t_{0}^{-}\right) x\left(t_{0}\right)+\frac{1}{2} \eta\left(t_{0}^{-}\right)=0
$$

from (18).

Now we introduce a new variable, namely incremental time, defined as $\Delta_{i} \triangleq t_{i+1}-t_{i} \quad i \in\{0,1, \cdots, N-1\}$. From (33) we have

$$
\begin{aligned}
p(t) & =e^{-A^{T}\left(t-t_{i}\right)} p\left(t_{i}^{+}\right) \quad t \in\left(t_{i}, t_{i+1}\right) \\
p\left(t_{i+1}^{+}\right) & =p\left(t_{i+1}^{-}\right)+\frac{1}{\lambda} C^{T}\left(r_{i+1}-C x\left(t_{i+1}\right)\right) \\
& =e^{-A^{T} \Delta_{i}} p\left(t_{i}^{+}\right)-\frac{1}{\lambda} C^{T} C x\left(t_{i+1}\right)+\frac{1}{\lambda} C^{T} r_{i+1}
\end{aligned}
$$

for $i \in\{0,1, \cdots, N-1\}$. From the dynamics of $x$ in (33), we have

$$
\begin{aligned}
x\left(t_{i+1}\right) & =e^{A\left(t_{i+1}-t_{i}\right)} x\left(t_{i}\right)-\int_{t_{i}}^{t_{i+1}} e^{A\left(t_{i+1}-\sigma\right)} B B^{T} p(\sigma) d \sigma \\
& =e^{A \Delta_{i}} x\left(t_{i}\right)-\int_{t_{i}}^{t_{i+1}} e^{A\left(t_{i+1}-\sigma\right)} B B^{T} e^{-A^{T}\left(\sigma-t_{i}\right)} p\left(t_{i}^{+}\right) d \sigma \\
& =e^{A \Delta_{i}} x\left(t_{i}\right)-e^{A \Delta_{i}}\left[\int_{t_{i}}^{t_{i+1}} e^{A\left(t_{i}-\sigma\right)} B B^{T} e^{-A^{T}\left(\sigma-t_{i}\right)} d \sigma\right] p\left(t_{i}^{+}\right) .
\end{aligned}
$$


From (36) and (37) we obtain the following matrix representation for forward-propagation of $x\left(t_{i}\right)$ and $p\left(t_{i}^{+}\right)$

$$
\left[\begin{array}{c}
x\left(t_{i+1}\right) \\
p\left(t_{i+1}^{+}\right)
\end{array}\right]=\left[\begin{array}{cc}
e^{A \Delta_{i}} & -e^{A \Delta_{i}} \mathbb{W}_{i} \\
-\frac{1}{\lambda} C^{T} C e^{A \Delta_{i}} & {\left[e^{-A^{T} \Delta_{i}}+\frac{1}{\lambda} C^{T} C e^{A \Delta_{i}} \mathbb{W}_{i}\right.}
\end{array}\right]\left[\begin{array}{c}
x\left(t_{i}\right) \\
p\left(t_{i}^{+}\right)
\end{array}\right]+\left[\begin{array}{c}
0 \\
\frac{1}{\lambda} C^{T}
\end{array}\right] r_{i+1}
$$

where $\mathbb{W}_{i}$ is defined as

$$
\begin{aligned}
\mathbb{W}_{i} & =\int_{t_{i}}^{t_{i+1}} e^{A\left(t_{i}-\sigma\right)} B B^{T} e^{-A^{T}\left(\sigma-t_{i}\right)} d \sigma \\
& =\int_{0}^{\Delta_{i}} e^{-A \tau} B B^{T} e^{-A^{T} \tau} d \tau \quad\left(\tau=\sigma-t_{i}\right) .
\end{aligned}
$$

From (39) it is apparent that the controllability Gramian $\mathbb{W}_{i}$ depends only on the inter-sample intervals, not explicitly on the sampling instances. Moreover,the Gramian is invertible as the underlying system (4) is controllable.

By defining a discrete time state vector as $z_{i}=\left[x^{T}\left(t_{i}\right) p^{T}\left(t_{i}^{+}\right)\right]^{T}$, (38) can be represented as the following discrete time system

$$
z_{i+1}=\Lambda_{i} z_{i}+\Gamma r_{i+1}
$$

where $\Lambda_{i}$ and $\Gamma$ are defined as

$$
\begin{aligned}
\Lambda_{i} & =\left[\begin{array}{cc}
e^{A \Delta_{i}} & -e^{A \Delta_{i}} \mathbb{W}_{i} \\
-\frac{1}{\lambda} C^{T} C e^{A \Delta_{i}} & {\left[e^{-A^{T} \Delta_{i}}+\frac{1}{\lambda} C^{T} C e^{A \Delta_{i}} \mathbb{W}_{i}\right.}
\end{array}\right] \\
\Gamma & =\left[\begin{array}{c}
0 \\
\frac{1}{\lambda} C^{T}
\end{array}\right] .
\end{aligned}
$$

Lemma 4.1: $\Lambda_{i}$ is invertible for any $i \in\{0,1, \cdots, N-1\}$.

Proof:

$$
\begin{aligned}
\Lambda_{i} & =\left[\begin{array}{cc}
e^{A \Delta_{i}} & -e^{A \Delta_{i}} \mathbb{W}_{i} \\
-\frac{1}{\lambda} C^{T} C e^{A \Delta_{i}} & {\left[e^{-A^{T} \Delta_{i}}+\frac{1}{\lambda} C^{T} C e^{A \Delta_{i}} \mathbb{W}_{i}\right.}
\end{array}\right] \\
& =\left[\begin{array}{cc}
I & 0 \\
-\frac{1}{\lambda} C^{T} C & I
\end{array}\right]\left[\begin{array}{cc}
e^{A \Delta_{i}} & -e^{A \Delta_{i}} \mathbb{W}_{i} \\
0 & e^{-A^{T} \Delta_{i}}
\end{array}\right] \\
& =M \Upsilon_{i}
\end{aligned}
$$

(41) gives a block LU-factorization for $\Lambda_{i}$ and both $M$ and $\Upsilon_{i}$ are invertible for any $i$.

Hence, $\Lambda_{i}$ is invertible for any $i$.

From (40) we obtain

$$
\begin{aligned}
z_{k} & =\left(\prod_{i=0}^{k-1} \Lambda_{i}\right)\left[\begin{array}{c}
x\left(t_{0}\right) \\
\frac{1}{\lambda} C^{T}\left(r_{0}-C x\left(t_{0}\right)\right)
\end{array}\right]+\sum_{i=1}^{k}\left(\prod_{j=i}^{k-1} \Lambda_{j}\right) \Gamma r_{i} \\
& =\left(\prod_{i=0}^{k-1} \Lambda_{i}\right)\left(\left[\begin{array}{c}
I \\
-\frac{1}{\lambda} C^{T} C
\end{array}\right] x\left(t_{0}\right)+\Gamma r_{0}\right)+\sum_{i=1}^{k}\left(\prod_{j=i}^{k-1} \Lambda_{j}\right) \Gamma r_{i} \\
& =\left(\prod_{i=0}^{k-1} \Lambda_{i}\right)\left[\begin{array}{c}
I \\
-\frac{1}{\lambda} C^{T} C
\end{array}\right] x\left(t_{0}\right)+\sum_{i=0}^{k}\left(\prod_{j=i}^{k-1} \Lambda_{j}\right) \Gamma r_{i}
\end{aligned}
$$

where $\prod$ represents left multiplication. As $p\left(t_{N}^{+}\right)=0, x\left(t_{0}\right)$ can be obtained by solving the following equation

$$
\left[\begin{array}{ll}
0 & I
\end{array}\right]\left(\prod_{i=0}^{N-1} \Lambda_{i}\right)\left[\begin{array}{c}
I \\
-\frac{1}{\lambda} C^{T} C
\end{array}\right] x\left(t_{0}\right)=-\left[\begin{array}{ll}
0 & I
\end{array}\right] \sum_{i=0}^{N}\left(\prod_{j=i}^{N-1} \Lambda_{j}\right) \Gamma r_{i} .
$$

From the way (43) has been obtained, it can be inferred that (43) is an alternative form of (18). Hence, it can be concluded from Theorem 3.4 that (43) yields a solution for almost any time index set $\left\{t_{i}\right\}_{i=0}^{N}$.

Once $x\left(t_{0}\right)$ is obtained by solving (43), the trajectory can be reconstructed using (33) and the jump conditions given by (34). 


\section{Ordinary Cross Validation to Choose an Optimal $\lambda$ for The Trajectory Smoothing Problem}

Ordinary cross validation (OCV) was first proposed by Allen (1974) in the context of regression [20] and by Grace Wahba and Wold (1975) for smoothing splines [21]. The main idea behind cross validation is to use a subset of the given dataset to obtain a parameter estimate and to use the rest of the data for performance validation under that particular value of the estimate. However, cross validation does not use one subset solely for one purpose (estimation or validation); it allows all the data to be used for both purposes. For instance, we can divide the data set into $m$ subsets; compute an estimate from all the subsets but one; and validate the estimate using the left-out subset. Then, we perform the estimation-validation after leaving out a different subset. This process is repeated $m$ times.

OCV uses "leaving-out-one" strategy, i.e. each data point is left out in turn and an estimate for the curve is derived from the rest of the data (by solving an optimization problem). Then the prediction error is computed at the left out data point and the prediction errors are summed to yield the ordinary cross validation cost. An optimal $\lambda$ minimizes the summed error.

Now we'll briefly discuss the ordinary cross validation procedure for the trajectory smoothing problem. Let $\left(x_{\lambda}^{k}(0), u_{\lambda}^{k}(\cdot)\right)$ be a minimizer of the following optimization problem:

$$
\underset{x\left(t_{0}\right), u}{\operatorname{Minimize}}\left(\sum_{\substack{j=0 \\ j \neq k}}^{N}\left\|r\left(t_{j}\right)-r_{j}\right\|^{2}+\lambda \int_{0}^{T} u^{T}(\sigma) u(\sigma) d \sigma\right)
$$

subject to the constraints given by (4). Then the ordinary cross validation cost $V_{0}(\lambda)$ is defined as

$$
V_{0}(\lambda)=\frac{1}{N+1} \sum_{k=0}^{N}\left\|r_{k}-r_{\lambda}^{k}\left(t_{k}\right)\right\|^{2}
$$

where $r_{\lambda}^{k}(\cdot)$ is obtained from (4), using $x_{\lambda}^{k}(0)$ as the initial condition and $u_{\lambda}^{k}(\cdot)$ as the input. Hence we choose the optimal value of the regularization parameter as

$$
\lambda^{*}=\underset{\lambda \in \mathbb{R}_{+}}{\operatorname{argmin}}\left(V_{0}(\lambda)\right) .
$$

For the problem under consideration, the special structure of the underlying dynamical system yields a nice form for the ordinary cross validation cost.

Now we solve the optimization problem (44) by following the path described in section IV of this article. By following the co-state approach we can conclude that the optimal trajectory will be a base integral curve of the associated Hamiltonian vector field, with suitable jump conditions on the co-state variables. It can be easily observed that the co-state variables are continuous at the left-out point, without any jump. Then with a little bit of algebra we can show that $x_{\lambda}^{k}(0)$, an optimal initial condition, will satisfy a modified form of (43), in particular

$$
\begin{aligned}
& {\left[\begin{array}{ll}
0 & I
\end{array}\right]\left(\prod_{i=k}^{N-1} M \Upsilon_{i}\right) \Upsilon_{k-1}\left(\prod_{i=0}^{k-2} M \Upsilon_{i}\right)\left[\begin{array}{c}
I \\
-\frac{1}{\lambda} C^{T} C
\end{array}\right] x_{\lambda}^{k}(0)} \\
& \quad=-\left[\begin{array}{ll}
0 & I
\end{array}\right] \sum_{i=0}^{k-1}\left(\prod_{j=k}^{N-1} M \Upsilon_{j}\right) \Upsilon_{k-1}\left(\prod_{j=i}^{k-2} M \Upsilon_{j}\right) \Gamma r_{i}-\left[\begin{array}{ll}
0 & I
\end{array}\right] \sum_{i=k+1}^{N}\left(\prod_{j=i}^{N-1} M \Upsilon_{j}\right) \Gamma r_{i}
\end{aligned}
$$

where $\Upsilon_{i}$ 's are obtained by factorization of $\Lambda_{i}$ 's, as mentioned in Lemma 4.1. Therefore the reconstruction error encountered at the $k$-th data point can be represented as

$$
\begin{aligned}
r_{k}-r_{\lambda}^{k}\left(t_{k}\right) & =r_{k}-C x_{\lambda}^{k}\left(t_{k}\right) \\
& =r_{k}-C\left[\begin{array}{l}
I \\
0
\end{array}\right]^{T} \Upsilon_{k-1}\left(\prod_{i=0}^{k-2} M \Upsilon_{i}\left[\begin{array}{c}
I \\
-\frac{1}{\lambda} C^{T} C
\end{array}\right] x_{\lambda}^{k}(0)+\sum_{i=0}^{k-1}\left(\prod_{j=i}^{k-2} M \Upsilon_{j}\right) \Gamma r_{i}\right)
\end{aligned}
$$

when we start the trajectory from $x_{\lambda}^{k}(0)$ and apply the optimal input $u_{\lambda}^{k}$. From (47) it is quite clear that $\lambda$ affects $x_{\lambda}^{k}(0)$ through $M, \Gamma$ and $\left[I-\frac{1}{\lambda} C^{T} C\right]$ and hence the reconstruction error is a vector of rational functions in $\lambda$. Now we can represent the cross validation cost, $V_{0}(\lambda)$, associated with this particular problem as

$$
V_{0}(\lambda)=\frac{1}{N+1} \sum_{k=0}^{N}\left(r_{k}^{T} r_{k}+\left(x_{\lambda}^{k}\left(t_{k}\right)\right)^{T} C^{T} C x_{\lambda}^{k}\left(t_{k}\right)-2 r_{k}^{T} C x_{\lambda}^{k}\left(t_{k}\right)\right) .
$$

As we have the (somewhat) closed form for the OCV cost, given by (49), we are now ready to write down the first order necessary condition for the optimality of the regularization parameter $\lambda$. We can easily check that the optimal value, $\lambda^{*}$, will 
satisfy the following first order condition

$$
\sum_{k=0}^{N}\left(\left(C x_{\lambda^{*}}^{k}\left(t_{k}\right)-r_{k}\right)^{T} C\left[\begin{array}{l}
I \\
0
\end{array}\right]^{T} \Upsilon_{k-1}\right) \times\left(\frac{\partial}{\partial \lambda}\left[\prod_{i=0}^{k-2} M \Upsilon_{i}\left[\begin{array}{c}
I \\
-\frac{1}{\lambda} C^{T} C
\end{array}\right] x_{\lambda}^{k}(0)+\sum_{i=0}^{k-1}\left(\prod_{j=i}^{k-2} M \Upsilon_{j}\right) \Gamma r_{i}\right]_{\lambda^{*}}\right)=0
$$

\section{Numerical ExAmples}

We generated two sets of synthetic data: (a) spiral on a sphere of radius 5 , and added i.i.d. Gaussian noise with mean $=$ 0 , and standard deviation $=0.15$; (b) circular helix on a cylinder of radius 5, and added i.i.d Gaussian noise with mean $=0$, and standard deviation $=0.05$. In each case the number of samples used was $N=201$. See Figures 1 and 2 for illustration. Reconstruction using the method of this paper (see figures) appears to produce satisfactory results as judged from the fit error normalized by the corresponding radius. In these numerical experiments the regularization/penalty parameter was determined by ordinary cross validation.

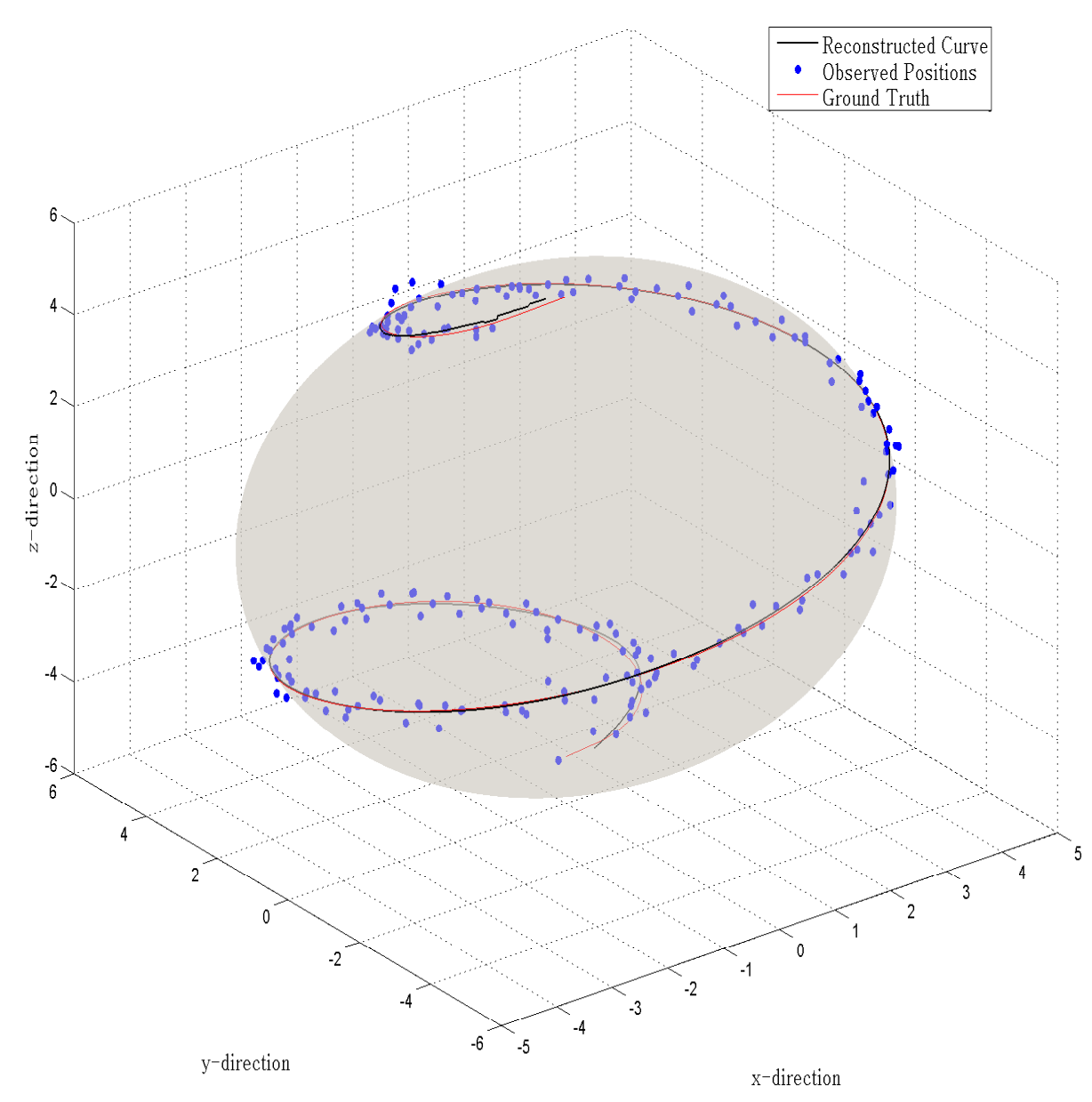

Fig. 1. Curve on a sphere $\left(\lambda^{*}: 4.50 \times 10^{-6}\right.$ and Avg. Fit Error/Radius: $\left.13.686 \times 10^{-3}\right)$.

\section{CONCLUSION}

Many areas of science and engineering (e.g. tracking individual trajectories of animal movement, motion capture in robotics) lead to the ill-posed inverse problem of extracting velocity and higher derivatives from a noisy finite sample of observed positions. Using a simple linear generative model for trajectories, this paper investigates a technique based on optimal control to obtain smoothed solutions to the inverse problem. The method is based on regularization by adding a penalty - the path integral of the square of jerk, to the fit error. The underlying cross validation technique for determining the regularization/penalty 


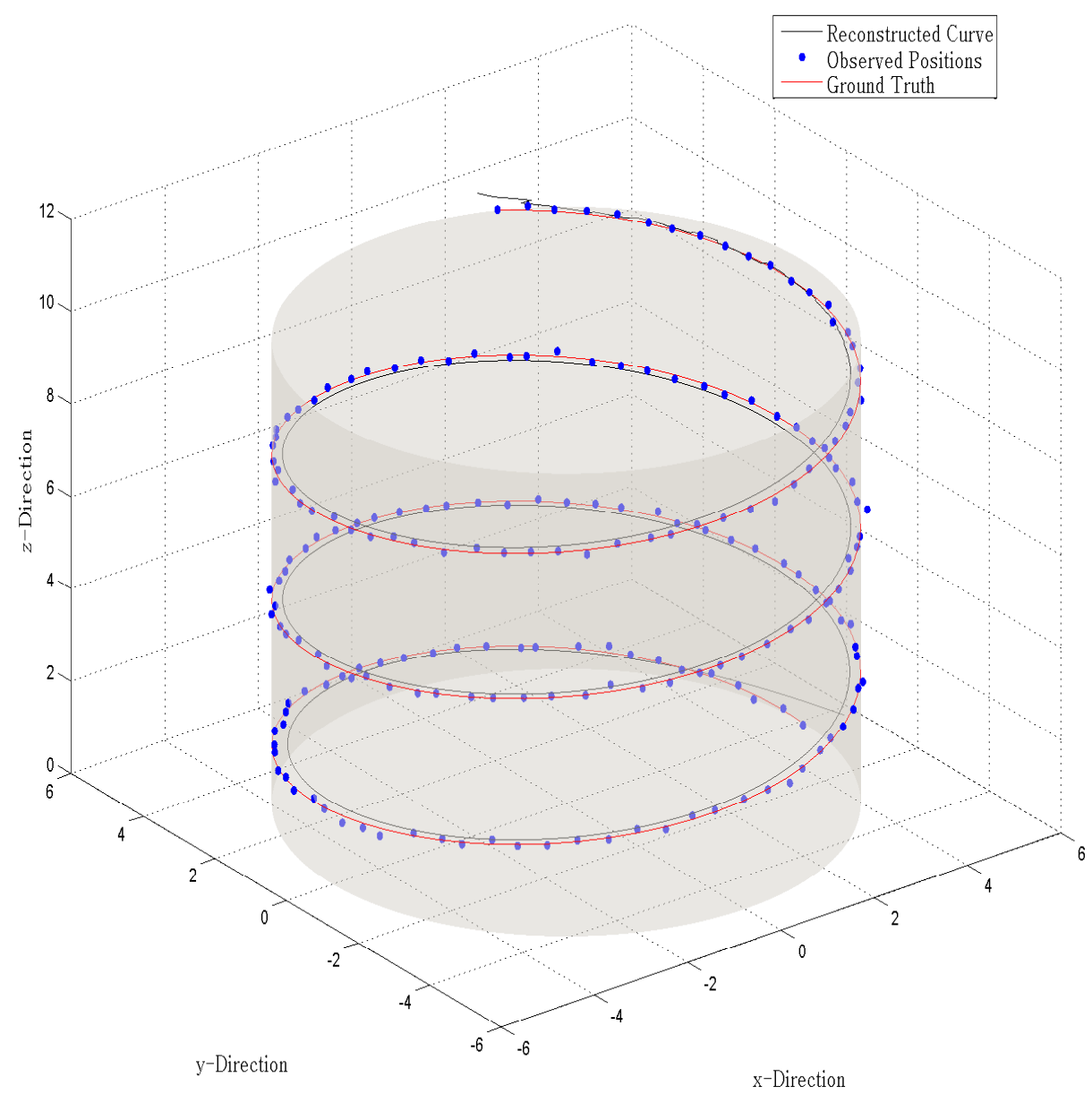

Fig. 2. Circular Helix $\left(\lambda^{*}: 3.75 \times 10^{-6}\right.$ and Avg. Fit Error/Radius: $\left.12.346 \times 10^{-3}\right)$.

parameter is also sketched out. The algorithm has been tested on synthetic data and is currently being applied to biological observations.

\section{APPENDIX A \\ SUPPLEMENTARY PROOFS}

Proposition A.1 (Matrix Inversion Lemma): For any four compatible matrices $E, F, G, H$, the following relationship holds true

$$
\left(E-F H^{-1} G\right)^{-1}=E^{-1}+E^{-1} F\left(H-G E^{-1} F\right)^{-1} G E^{-1}
$$

whenever $E$ and $H$ are invertible.

Proof: We will be using the following two matrix identities to prove the matrix inversion lemma

$$
\begin{aligned}
(I+P)^{-1} & =I-(I+P)^{-1} P \\
(I+P Q)^{-1} P & =P(I+Q P)^{-1} .
\end{aligned}
$$

Now,

$$
\begin{aligned}
\left(E-F H^{-1} G\right)^{-1} & =\left[E\left(I-E^{-1} F H^{-1} G\right)\right]^{-1} \\
& =\left(I-E^{-1} F H^{-1} G\right)^{-1} E^{-1} \\
& =\left[I+\left(I-E^{-1} F H^{-1} G\right)^{-1} E^{-1} F H^{-1} G\right] E^{-1}
\end{aligned}
$$




$$
\begin{aligned}
& =E^{-1}+\left(I-E^{-1} F H^{-1} G\right)^{-1} E^{-1} F H^{-1} G E^{-1} \\
& =E^{-1}+E^{-1} F\left(I-H^{-1} G E^{-1} F\right)^{-1} H^{-1} G E^{-1} \\
& =E^{-1}+E^{-1} F\left[H^{-1}\left(H-G E^{-1} F\right]^{-1} H^{-1} G E^{-1}\right. \\
& =E^{-1}+E^{-1} F\left(H-G E^{-1} F\right)^{-1} G E^{-1} .
\end{aligned}
$$

Theorem A.2: Consider a Riccati equation of the form

$$
\begin{aligned}
\dot{K}(t) & =-K(t) A-A^{T} K(t)+K(t) B B^{T} K(t) \\
K(T) & =Q .
\end{aligned}
$$

It has a symmetric, positive semi-definite solution $K(t)$ for any $t \leq T$ and controllable $[A, B]$ whennever the terminal condition $Q$ is symmetric, positive semi-definite $\left(Q=Q^{T} \geq 0\right)$.

Proof: The adjoint system corresponding to the Riccati equation (A53) is

$$
\frac{d}{d t}\left[\begin{array}{l}
\eta_{1} \\
\eta_{2}
\end{array}\right]=\left[\begin{array}{cc}
A & -B B^{T} \\
0 & -A^{T}
\end{array}\right]\left[\begin{array}{l}
\eta_{1} \\
\eta_{2}
\end{array}\right]
$$

and the associated transition matrix can be represented as

$$
\left[\begin{array}{ll}
\phi_{11} & \phi_{12} \\
\phi_{21} & \phi_{22}
\end{array}\right](t, T)=\phi(t, T)=\left[\begin{array}{cc}
e^{A(t-T)} & e^{A(t-T)} \mathbb{G}(t, T) \\
0 & e^{-A^{T}(t-T)}
\end{array}\right]
$$

where,

$$
\mathbb{G}(t, T) \triangleq-\int_{T}^{t} e^{A(T-\sigma)} B B^{T} e^{A^{T}(T-\sigma)} d \sigma
$$

is positive definite for any $t<T$ because of controllability of the pair $[A, B]$.

Hence the solution for (A53) can be represented as,

$$
\begin{aligned}
K(t) & =\left[\phi_{21}(t, T)+\phi_{22}(t, T) Q\right]\left[\phi_{11}(t, T)+\phi_{12}(t, T) Q\right]^{-1} \\
& =e^{-A^{T}(t-T)} Q\left[e^{A(t-T)}(I+\mathbb{G}(t, T) Q)\right]^{-1} \\
& =e^{-A^{T}(t-T)} Q(I+\mathbb{G}(t, T) Q)^{-1} e^{-A(t-T)} .
\end{aligned}
$$

Now by applying the matrix inversion lemma, and by letting $E=I, F=-I, G=Q$ and $H=(\mathbb{G}(t, T))^{-1}$ we obtain

$$
(I+\mathbb{G}(t, T) Q)^{-1}=I-\left((\mathbb{G}(t, T))^{-1}+Q\right)^{-1} Q
$$

As $\mathbb{G}(t, T)>0$ for any $t<T$, its inverse is also positive definite for any $t<T$. Then positive definiteness of $\left[(\mathbb{G}(t, T))^{-1}+\right.$ $Q]^{-1}$ is directly implied from the fact that the terminal condition $Q$ is positive semi-definite.

By defining $\mathbb{M}(t) \triangleq(\mathbb{G}(t, T))^{-1}$, we have

$$
K(t)=e^{-A^{T}(t-T)} Q\left[Q-Q[\operatorname{M}(t)+Q]^{-1} Q\right] Q e^{-A(t-T)} .
$$

As $\mathbb{I M}>0$ (implicit dependency on time $t$ is not shown for the sake of clarity) and $Q=Q^{T} \geq 0$, there exists a non-singular matrix $P$ such that

$$
\begin{aligned}
& P^{T} Q P=\Lambda \\
& P^{T} \mathbb{M} P=I
\end{aligned}
$$

where $\Lambda$ is a diagonal matrix with non-negative entries. With the above expressions from simulataneous diagonalization, we have

$$
Q-Q[\mathrm{M}+Q]^{-1} Q=\left(P^{T}\right)^{-1}\left[\Lambda-\Lambda(I+\Lambda)^{-1} \Lambda\right] P^{-1}
$$


Now, by assuming $\Lambda=\operatorname{diag}\left(\lambda_{1}, \cdots, \lambda_{n}\right), \lambda_{i} \geq 0$, we obtain

$$
\begin{aligned}
& (I+\Lambda)^{-1}=\operatorname{diag}\left(\frac{1}{1+\lambda_{1}}, \cdots, \frac{1}{1+\lambda_{n}}\right) \\
\Rightarrow & \Lambda(I+\Lambda)^{-1} \Lambda=\operatorname{diag}\left(\frac{\lambda_{1}^{2}}{1+\lambda_{1}}, \cdots, \frac{\lambda_{n}^{2}}{1+\lambda_{n}}\right) \\
\Rightarrow & \Lambda-\Lambda(I+\Lambda)^{-1} \Lambda=\operatorname{diag}\left(\frac{\lambda_{1}}{1+\lambda_{1}}, \cdots, \frac{\lambda_{n}^{2}}{1+\lambda_{n}}\right)
\end{aligned}
$$

Therefore $\Lambda-\Lambda(I+\Lambda)^{-1} \Lambda$ is a postive semi-definite diagonal matrix, and hence from (A54), $Q-Q[\mathrm{M}+Q]^{-1} Q$ is a symmetric positive semi-definite matrix.

Hence, $K(t)$ is symmetric, postive semi-definite for any $t<T$.

\section{REFERENCES}

[1] J. Gautrais, C. Jost, M. Soria, A. Campo, S. Motsch, R. Fournier, S. Blanco, and G. Theraulaz, "Analyzing fish movement as a persistent turning walker," Journal of Mathematical Biology, vol. 58, no. 3, pp. 429-445, March 2009.

[2] M. Ballerini, N. Cabibbo, R. Candelier, A. Cavagna, E. Cisbani, I. Giardina, A. Orlandi, G. Parisi, A. Procaccini, M. Viale, and V. Zdravkovic, "Empirical investigation of starling flocks: A benchmark study in collective animal behaviour," Animal Behaviour, vol. 76, pp. 201-215, 2008.

[3] C. Chiu, P. V. Reddy, W. Xian, P. S. Krishnaprasad, and C. F. Moss, "Effects of competitive prey capture on flight behavior and sonar beam pattern in paired big brown bats, eptesicus fuscus." Journal of Experimental Biology, vol. 213, no. 19, pp. 3348-3356, 2010.

[4] R. M. Olberg, A. H. Worthington, and K. R. Venator, "Prey pursuit and interception in dragonflies," Journal of Comparative Physiology A, vol. 186, no. 2, pp. 155-162, February 2000.

[5] G. Wahba, Spline Models for Observational Data, ser. CBMS-NSF Regional Conference Series in Applied Mathematics. Philadelphia, PA: SIAM, 1990, vol. 59.

[6] K. Ghose, T. K. Horiuchi, P. S. Krishnaprasad, and C. F. Moss, "Echolocating bats use a nearly time-optimal strategy to intercept prey," PLoS Biology, vol. 4, no. 5, pp. 865-873, May 2006.

[7] R. L. Bishop, "There is more than one way to frame a curve," The American Mathematical Monthly, vol. 82, no. 3, pp. $246-251$, 1975.

[8] E. W. Justh and P. S. Krishnaprasad, "Natural frames and interacting particles in three dimensions," in Proceedings of 44th IEEE Conference on Decision and Control and 2005 European Control Conference (CDC-ECC '05), Seville, Spain, 2005, pp. 2841 - 2846.

[9] P. Reddy, Steering Laws for Pursuit. Master's Thesis: University of Maryland, College Park, 2007.

[10] P. V. Reddy, E. W. Justh, and P. S. Krishnaprasad, "Motion camouflage with sensorimotor delay," in Proceedings of 46th IEEE Conference on Decision and Control, New Orleans, LA, 2007, pp. $1660-1665$.

[11] V. Jurdjevic, Geometric Control Theory, ser. Cambridge Studies in Advanced Mathematics. Cambridge: Cambridge University Press, 1997, vol. 52.

[12] E. W. Justh and P. S. Krishnaprasad, "Optimal natural frames," Communications in Information and Systems, vol. 11, no. 1, pp. 17-34, 2011.

[13] M. Egerstedt and C. Martin, Control Theoretic Splines: Optimal Control, Statistics, and Path Planning. Princeton, NJ: Princeton University Press, Feb. 2010.

[14] T. Flash and N. Hogan, "The coordination of arm movements: An experimentally confirmed mathematical model," The Journal of Neuroscience, vol. 5, no. 7, pp. 1688-1703, July 1985.

[15] E. Todorov and M. Jordan, "Smoothness maximization along a predefined path accurately predicts the speed profiles of complex arm movements," Journal of Neurophysiology, vol. 80, no. 2, pp. 696-714, 1998.

[16] R. Brockett, Finite Dimensional Linear Systems. New York: Wiley, 1970.

[17] L. M. Silverman and H. E. Meadows, "Controllability and observability in time-variable linear systems," SIAM Journal on Control and Optimization, vol. 5, no. 1, pp. 64-73, 1967.

[18] A. Savitzky and M. J. E.Golay, "Smoothing and differentiation of data by simplified least squares procedures," Analytical Chemistry, vol. 36, no. 8, pp. 1627-1639, July 1964.

[19] R. W. Schafer, "What is a savitzky-golay filter? [lecture notes]," IEEE Signal Processing Magazine, vol. 28, no. 4, pp. 111 -117, July 2011.

[20] D. M. Allen, "The relationship between variable selection and data augmentation and a method for prediction," Technometrics, vol. 16, no. 1, pp. 125-127, February 1974.

[21] G. Wahba and S. Wold, "A completely automatic french curve: Fitting spline functions by cross-validation," Communications in Statistics, vol. 4, no. 1, pp. 1-17, 1975. 